\title{
Lie theory for symmetric Leibniz algebras
}

\author{
Mamuka Jibladze $^{1} \cdot$ Teimuraz Pirashvili $^{2}$
}

Received: 6 May 2019 / Accepted: 10 September 2019 / Published online: 5 October 2019

(C) The Author(s) 2019

\begin{abstract}
Lie algebras and groups equipped with a multiplication $\mu$ satisfying some compatibility properties are studied. These structures are called symmetric Lie $\mu$-algebras and symmetric $\mu$-groups respectively. An equivalence of categories between symmetric Lie $\mu$-algebras and symmetric Leibniz algebras is established when 2 is invertible in the base ring. The second main result of the paper is an equivalence of categories between simply connected symmetric Lie $\mu$-groups and finite dimensional symmetric Leibniz algebras.
\end{abstract}

Keywords Lie algebras · Leibniz algebras $\cdot$ Lie groups $\cdot$ Algebraic theories

Mathematics Subject Classification 17A32 · 22E60

\section{Introduction}

One of the leading motivations for introducing Leibniz algebras by Loday was an intriguing possibility to define some mythical objects, which he named coquecigrues (after Rabelais). Those should stand in the same relation to groups, as Leibniz algebras are to Lie algebras. See [4, Section 11] for details, or [5, Section 5] for further details and possible consequences for algebraic K-theory, etc.

Recently the second author looked into his very old file of papers and discovered our notes written in 1995 and entitled "The first step to coquecigrue: the grin", as well as unpublished notes by Ronco [9] written about the same time, where among other results our Corollary 6 is stated.

Dedicated to the memory of Jean-Louis Loday.

Communicated by Soso Gubeladze.

\Teimuraz Pirashvili

tp59@leicester.ac.uk

1 Razmadze Mathematical Institute, Tbilisi, Georgia

2 Department of Mathematics, University of Leicester, Leicester, UK 
Reading these notes after 24 years, we think they still are of some interest. The present paper essentially consists of those notes of ours, with the addition of present Sect. 6.

The aim of this work is to extend the classical correspondence between Lie groups and Lie algebras to a bit wider class, namely between so called Lie $\mu$-groups and symmetric Leibniz algebras.

In Sect. 2 we introduce symmetric Leibniz algebras and state some of their properties that we will need.

In Sect. 3 we define the closely related symmetric Lie $\mu$-algebras, and in Sect. 4 we prove that upon inverting 2 they become equivalent to symmetric Leibniz algebras (Theorem 11).

In Sect. 5, group side counterparts of these structures enter, under the name of symmetric $\mu$-groups ( $\Sigma \mu$-groups for short). We exhibit there some of their properties parallel to those for symmetric Leibniz algebras.

In Sect. 6 we consider the straightforward analogs of Lie groups for $\Sigma \mu$-groups and prove the analog of the classical equivalence between Lie algebras and simply connected Lie groups-Corollary 19 describes an equivalence between the category of finite dimensional symmetric Leibniz algebras and simply connected Lie $\Sigma \mu$-groups.

Section 7 recasts our results in terms of the formalism of [1]. We describe the theory of symmetric Leibniz algebras as a linear extension of the theory of Lie algebras. We also describe theories of symmetric Leibniz algebras and symmetric $\mu$-groups as pullbacks of diagrams of simpler theories.

Let us finish the introduction with acknowledging very useful advice of the referee which helped to improve the paper a lot.

\section{Symmetric Leibniz algebras}

We fix a commutative ring K. All modules and tensor products are taken over K. The category of modules is denoted by $\mathfrak{M D D}$ and the category of Lie algebras by $\mathfrak{L} \mathfrak{I}$. We identify modules with abelian Lie algebras, so we have the inclusion $\mathfrak{M O D} \subset \mathfrak{L} \mathfrak{I E}$.

Leibniz algebras are a generalization of Lie algebras. Importance of these algebras was realized by Jean-Louis Loday, see [3, Section 10.6]. Recall that a (right) Leibniz algebra is a module $\mathcal{L}$, equipped with an operation $[-,-]: \mathcal{L} \otimes \mathcal{L} \rightarrow \mathcal{L}$ such that the right Leibniz identity holds:

$$
[x,[y, z]]=[[x, y], z]-[[x, z], y] .
$$

We refer the reader to [5-7,10] for more on Leibniz algebras, Leibniz homology, Leibniz representations and conjectures about coquecigrue. For other conjectures for Leibniz algebras and Leibniz homology see [12,13].

The category of Leibniz algebras is denoted by $\mathfrak{L} \mathfrak{B}$. Recall that in any Leibniz algebra one has identities

$$
[x,[y, y]]=0, \quad[x,[y, z]]+[x,[z, y]]=0,
$$


which are direct consequences of the right Leibniz identity (1).

An exact sequence

$$
0 \rightarrow \mathcal{L}_{1} \stackrel{i}{\rightarrow} \mathcal{L} \stackrel{p}{\rightarrow} \mathcal{L}_{2} \rightarrow 0
$$

of Leibniz algebras and Leibniz algebra homomorphisms is called abelian if $\mathcal{L}_{1}$ is an abelian Leibniz algebra. Moreover it is called central if $\operatorname{Im}(i)$ is a central subalgebra of $\mathcal{L}$, that is

$$
[i(a), x]=0=[x, i(a)]
$$

hold for all $x \in \mathcal{L}$ and $a \in \mathcal{L}_{1}$.

Let $\mathcal{L}$ be a Leibniz algebra. The submodule of $\mathcal{L}$ generated by elements of the form $[x, x], x \in \mathcal{L}$ is denoted by $\mathcal{L}^{\text {ann }}$. It is a two-sided ideal of $\mathcal{L}[6]$. The quotient $\mathcal{L} / \mathcal{L}^{\text {ann }}$ is a Lie algebra, denoted by $\mathcal{L}_{\text {Lie }}$ and called the Liezation of $\mathcal{L}$. The functor $\mathcal{L} \mapsto \mathcal{L}_{\text {Lie }}$ is the left adjoint to the inclusion $\mathfrak{L} \mathfrak{I} \subset \mathfrak{L} \mathfrak{B}$ [6]. The inclusion $\mathfrak{M} \mathfrak{O} \mathfrak{D} \subset \mathfrak{L} \mathfrak{B}$ also has a left adjoint functor, which is given by $\mathcal{L} \mapsto \mathcal{L}_{\text {ab }}:=\mathcal{L} /[\mathcal{L}, \mathcal{L}]$. The module $\mathcal{L}_{\text {ab }}$ is known as the abelization of $\mathcal{L}$.

Lemma 1 Let $\mathcal{L}$ be a Leibniz algebra satisfying the left Leibniz identity:

$$
[[x, y], z]=[x,[y, z]]-[y,[x, z]] .
$$

\section{Then one has}

i) $[[x, x], y]=0$,

ii) $[[x, y], z]+[[y, x], z]=0$,

iii) $[[x, y], z]+[z,[x, y]]=0$,

iv) $[[y, z], x]=[[x, z], y]-[[x, y], z]$,

v) $[[[a, b], c], d]=[[[d, c], a], b]-[[[d, c], b], a]-[[[d, a], b], c]+[[[d, b], a], c]$, vi) $2[[x, y],[x, y]]=0$.

Proof Take $x=y$ in the equality (3) to obtain i). The identity ii) is a formal consequence of i). Relation iii) can be obtained by addition of the identities (1) and (3). To show iv) one uses iii) and then the equality (1):

$$
[[y, z], x]=-[[x,[y, z]]=[[x, z], y]-[[x, y], z] .
$$

Relation v) can be deduced from iv), if one puts $y=[a, b], z=c, x=d$ and then uses the equality (1):

$$
\begin{aligned}
{[[[a, b], c], d] } & =[[d, c],[a, b]]-[[d,[a, b]], c] \\
& =[[[d, c], a], b]-[[[d, c], b], a]-[[[d, a], b], c]+[[[d, b], a], c] .
\end{aligned}
$$

In order to prove vi) we take $a=d=x$ and $b=c=y$ in v):

$[[[x, y], y], x]=[[x, y], x], y]-[[x, y], y], x]-[[[x, x], y], y]+[[[x, y], x], y]$. 
By the identity i) the third summation vanishes, and we get

$$
2[[[x, y], y], x]]=2[[[x, y], x], y] .
$$

Now vi) can be obtained using the identity (1):

$$
2[[x, y],[x, y]]=2[[[x, y], x], y]-2[[[x, y], y], x]=0 .
$$

Definition 2 A Leibniz algebra is symmetric, if it satisfies the left Leibniz identity (3) and

$$
[[x, y],[x, y]]=0 .
$$

By definition we have all identities proved in Lemma 1. Moreover, by vi) of the same Lemma, if two-torsion in $\mathcal{L}$ is zero, then the condition (4) in Definition 2 is redundant.

Let $\mathfrak{S} \mathfrak{L} \mathfrak{B}$ be the category of symmetric Leibniz algebras. It is obvious that Lie algebras are exactly those symmetric Leibniz algebras for which the identity $[x, x]=0$ holds. In particular we have inclusions $\mathfrak{L} \Im \mathfrak{E} \subset \mathfrak{S} \mathfrak{L} \mathfrak{B} \subset \mathfrak{L} \mathfrak{B}$.

Let $\mathcal{L}$ be a symmetric Leibniz algebra. Then by Lemma 1 we have

$$
\left[\mathcal{L}, \mathcal{L}^{\mathrm{ann}}\right]=0=\left[\mathcal{L}^{\mathrm{ann}}, \mathcal{L}\right]
$$

Thus one has a central extension of Leibniz algebras

$$
0 \rightarrow \mathcal{L}^{\text {ann }} \rightarrow \mathcal{L} \rightarrow \mathcal{L}_{\text {Lie }} \rightarrow 0
$$

We will need the second divided power $\Gamma^{2} V$ of a module $V$ [11]. Recall that $\Gamma^{2} V$ is generated as a module by elements of the form $x^{[2]}, x \in V$. These generators must satisfy the following relations

$$
\begin{aligned}
& (x+y+z)^{[2]}-(x+y)^{[2]}-(x+z)^{[2]}-(y+z)^{[2]}+x^{[2]}+y^{[2]}+z^{[2]}=0, \\
& (k x)^{[2]}=k^{2} x^{[2]} .
\end{aligned}
$$

Here $k \in \mathrm{K}$ and $x, y, z \in V$. We set

$$
x \cdot y=(x+y)^{[2]}-x^{[2]}-y^{[2]} .
$$

In this notations we have

$$
x \cdot x=2 x^{[2]} \text {. }
$$

It follows from the definition that $x \cdot y$ is bilinear and symmetric on $x$ and $y$. If $V$ is a free module, then $\Gamma^{2} V$ fits in the short exact sequence

$$
0 \rightarrow \Gamma^{2} V \stackrel{i}{\rightarrow} V^{\otimes 2} \rightarrow \Lambda^{2} V \rightarrow 0
$$


where $i\left(x^{[2]}\right)=x \otimes x$. Observe that $i(x \cdot y)=x \otimes y+y \otimes x$.

Recall also that if 2 is invertible in $\mathrm{K}$, then the map $V^{\otimes 2} \rightarrow \Gamma^{2}(V)$ given by $x \otimes y \rightarrow x \cdot y$ yields an isomorphism $\operatorname{Sym}^{2}(V) \cong \Gamma^{2}(V)$. Here and elsewhere $\operatorname{Sym}^{2}$ denotes the second symmetric power.

Lemma 3 i) Let $\mathcal{L}$ be a symmetric Leibnizalgebra. Then there is a well-defined linear map $\sigma: \Gamma^{2}\left(\mathcal{L}_{\mathrm{ab}}\right) \rightarrow \mathcal{L}$ given by

$$
\sigma\left(\bar{x}^{[2]}\right)=[x, x] .
$$

ii) One has an exact sequence

$$
\Gamma^{2}\left(\mathcal{L}_{\mathrm{ab}}\right) \stackrel{\sigma}{\rightarrow} \mathcal{L} \rightarrow \mathcal{L}_{\text {Lie }} \rightarrow 0
$$

of Leibniz algebras and Leibniz algebra homomorphisms, where $\Gamma^{2}\left(\mathcal{L}_{\mathrm{ab}}\right)$ is considered as an abelian Leibniz algebra. Moreover $\operatorname{Im}(\sigma)$ is a central subalgebra of $\mathcal{L}$.

Proof i) For any $x, y, z \in \mathcal{L}$ we have

$$
\begin{aligned}
{[x+[y, z], x+[y, z]] } & =[x, x]+[x,[y, z]]+[[y, z], x]+[[y, z],[y, z]] \\
& =[x, x] .
\end{aligned}
$$

Here we used iii) and vi) of Lemma 1. Thus $\sigma$ is well-defined.

ii) Comparing the definitions we see that $\operatorname{Im}(\sigma)=\mathcal{L}^{\text {ann }}$ and hence the result.

Recall that a Leibniz algebra $\mathcal{L}$ is perfect if $\mathcal{L}=[\mathcal{L}, \mathcal{L}]$.

Corollary 4 Any perfect symmetric Leibniz algebra is a Lie algebra.

Proof In this case $\mathcal{L}_{\mathrm{ab}}=0$. Hence $\mathcal{L} \rightarrow \mathcal{L}_{\text {Lie }}$ is an isomorphism thanks to the part ii) of Lemma 3.

Recall that [15] the free Lie algebra Lie $(V)$ generated by a free module $V$ has a natural grading

$$
\operatorname{Lie}(V)=\operatorname{Lie}_{1}(V) \oplus \operatorname{Lie}_{2}(V) \oplus \operatorname{Lie}_{3}(V) \oplus \cdots,
$$

where $\operatorname{Lie}_{n}(V)$ is spanned by all $n$-fold commutators of elements of $V$. For $n=1$ and $n=2$ we have $\operatorname{Lie}_{1}(V)=V$ and $\operatorname{Lie}_{2}(V)=\Lambda^{2} V$.

Recall also that [6] the free Leibniz algebra Leib $(V)$ generated by a module $V$ is also graded:

$$
\operatorname{Leib}(V)=V \oplus V^{\otimes 2} \oplus V^{\otimes 3} \oplus \cdots,
$$

where the bracket on Leib $(V)$ is uniquely defined by the rule:

$$
[\omega, v]=\omega \otimes v
$$


Here $\omega \in V^{\otimes n}, n \geq 1, v \in V$. In particular the map

$$
\pi_{n}: V^{\otimes n} \rightarrow \operatorname{Lie}_{n}(V)
$$

given by

$$
v_{1} \otimes \cdots \otimes v_{n} \mapsto\left[\left[\left[v_{1}, v_{2}\right], \cdots v_{n-1}\right], v_{n}\right]
$$

defines a surjective graded homomorphism of Leibniz algebras

$$
\pi: \operatorname{Leib}(V) \rightarrow \operatorname{Lie}(V)
$$

which clearly induces an isomorphism $(\operatorname{Leib}(V))_{\text {Lie }} \cong \operatorname{Lie}(V)$. It is also clear that the map $\pi$ is an isomorphism in degree 1 .

Our next goal is to describe the free symmetric Leibniz algebra SymLeib $(V)$ generated by $V$. Clearly the homomorphism $\pi$ has the following decomposition

$$
\operatorname{Leib}(V) \stackrel{\varpi^{1}}{\longrightarrow} \operatorname{SymLeib}(V) \stackrel{\varpi^{2}}{\longrightarrow} \operatorname{Lie}(V),
$$

where $\varpi^{1}$ and $\varpi^{2}$ are surjective Leibniz algebra homomorphisms.

Proposition 5 Let $V$ be a free module. Then for $\mathcal{L}=\operatorname{SymLeib}(V)$, one has a central extension of Leibniz algebras:

$$
0 \rightarrow \Gamma^{2}\left(\mathcal{L}_{\mathrm{ab}}\right) \stackrel{\sigma}{\rightarrow} \mathcal{L} \rightarrow \mathcal{L}_{\text {Lie }} \rightarrow 0 .
$$

Proof Since the defining relations of Leibniz and symmetric Leibniz algebras are of degree 3, the free Leibniz and symmetric Leibniz algebras have the same components in degree 1 and 2, which are respectively $V=\mathcal{L}_{\text {ab }}$ and $V^{\otimes 2}$. Thus injectivity of $\sigma$ follows from the exact sequence (5). The rest follows from Lemma 3.

Corollary 6 The free symmetric Leibniz algebra SymLeib $(V)$ is a graded module

$$
\operatorname{SymLeib}(V)=\bigoplus_{n \geq 0} \operatorname{SymLeib}_{n}(V) \text {. }
$$

Moreover, the map

$$
\varpi_{n}^{1}: V^{\otimes n} \rightarrow \operatorname{SymLeib}_{n}(V)
$$

is an isomorphism when $n=1,2$, while the map

$$
\varpi_{n}^{2}: \operatorname{SymLeib}_{n}(V) \rightarrow \operatorname{Lie}_{n}(V)
$$

is an isomorphism, when $n \geq 3$. Thus we have

$$
\operatorname{SymLeib}(V)=V \oplus V^{\otimes 2} \oplus \mathrm{Lie}_{3} V \oplus \mathrm{Lie}_{4} V \oplus \cdots .
$$


This result in the same period (i.e. around 1995) was obtained independently by Ronco [9] when $\mathrm{K}$ is a field of characteristic $\neq 2$.

\section{Symmetric Lie $\mu$-algebras}

We now introduce the notion of symmetric Lie $\mu$-algebra, or, for short, Lie $\Sigma \mu$ algebra. The strange terminology stems from the fact that we actually aim at general Lie $\mu$-algebras, to be investigated in [2], of which the symmetric Lie $\mu$-algebras are a particular case.

Definition 7 A symmetric Lie $\mu$-algebra is a pair $(\mathfrak{m}, \mu)$, where $\mathfrak{m}$ is a Lie algebra and $\mu: \mathfrak{m} \otimes \mathfrak{m} \rightarrow \mathfrak{m}$ is a multiplication, such that the following identities hold

i) $x y=y x$,

ii) $x(y z)=0=(x y) z$,

iii) $x\{y, z\}=0$,

iv) $\{x y, z\}=0$.

Here $\{-,-\}$ denotes the Lie bracket on $\mathfrak{m}$ and $x y=\mu(x \otimes y)$.

The first two identities show that the pair $(\mathfrak{m}, \mu)$ is a commutative, associative nilpotent algebra of class two. Conversely, any such algebra can be seen as a Lie $\Sigma \mu$-algebra with trivial bracket.

Any Lie algebra can be considered as a Lie $\Sigma \mu$-algebra with zero multiplication $x y=0$. In particular any module has the structure of a Lie $\Sigma \mu$-algebra with trivial bracket and trivial multiplication. Such Lie $\Sigma \mu$-algebras are called abelian Lie $\Sigma \mu$ algebras.

A Lie $\Sigma \mu$-ideal of a Lie $\Sigma \mu$-algebra $\mathfrak{m}$ is a submodule $\mathfrak{a}$ such that $[\mathfrak{m}, \mathfrak{a}] \subset \mathfrak{a}$ and $\mathfrak{m a} \subset \mathfrak{a}$. Moreover $\mathfrak{a}$ is a central $\Sigma \mu$-ideal if $[\mathfrak{m}, \mathfrak{a}]=0=\mathfrak{m a}$.

It follows from iv) of Definition 7 that submodule $\operatorname{Im}(\mu)$ is a central ideal of $\mathfrak{m}$. The quotient algebra is denoted by $\mathfrak{g}=\mathfrak{m}_{\text {Lie }}$ and is called the Liezation of $\mathfrak{m}$. The abelization of the Lie $\Sigma \mu$-algebra $\mathfrak{m}$ is the module $\mathfrak{g}_{\mathrm{ab}}$ and is denoted by $\mathfrak{m}_{\mathrm{AB}}$ in order to distinguish it from $\mathfrak{m}_{\mathrm{ab}}$ which is the abelization of the underlying Lie algebra. The last object has a canonical structure of a commutative, associative nilpotent algebra of class two, because by the identity iii) Lie commutator $[\mathfrak{m}, \mathfrak{m}]$ is an ideal of the underlying commutative algebra.

Lemma 8 For a $\Sigma \mu$-algebra $\mathfrak{m}$ the product $\mu: \mathfrak{m} \otimes \mathfrak{m} \rightarrow \mathfrak{m}$ factors through $\operatorname{Sym}^{2}\left(\mathfrak{m}_{\mathrm{AB}}\right)$ yielding an exact sequence of $\Sigma \mu$-algebras

$$
\operatorname{Sym}^{2}\left(\mathfrak{m}_{\mathrm{AB}}\right) \stackrel{\mu^{\prime}}{\rightarrow} \mathfrak{m} \rightarrow \mathfrak{g} \rightarrow 0
$$

where $\mathfrak{g}=\mathfrak{m}_{\text {Lie }}, \mu^{\prime}(\bar{x} \odot \bar{y})=x y$ and $\operatorname{Sym}^{2}\left(\mathfrak{m}_{\mathrm{AB}}\right)$ is considered as abelian Lie $\Sigma \mu$ algebra. Here $\bar{x}$ denotes the class of $x \in \mathfrak{m}$ in $\mathfrak{m}_{\mathrm{AB}}$. Furthermore $\operatorname{Im}\left(\mu^{\prime}\right)$ is a central $\Sigma \mu$-ideal of $\mathfrak{m}$. 
Proof By our construction we have an exact sequence:

$$
\mathfrak{m} \otimes \mathfrak{m} \stackrel{\mu}{\longrightarrow} \mathfrak{m} \rightarrow \mathfrak{g} \rightarrow 0
$$

Let us use identities of Definition 7. By the identity i) the map $\mu$ factors through $\operatorname{Sym}^{2}(\mathfrak{m})$. Next, by the identity ii) it further factors through $\operatorname{Sym}^{2}\left(\mathfrak{m}_{\text {Lie }}\right)$. It follows from the identities iii) that this map factors through the second symmetric power of abelization $\mathfrak{m}_{A B}$ and hence the result.

A Lie algebra $\mathfrak{g}$ is called $\Sigma \mu$-rigid if the only $\Sigma \mu$-algebra structure on it is the trivial one: $x y=0, x, y \in \mathfrak{g}$.

Corollary 9 Let $\mathfrak{m}$ be a Lie algebra, $\mathfrak{z}$ be the center of $\mathfrak{m}$ and $\mathfrak{h}=\mathfrak{m} /[\mathfrak{m}, \mathfrak{m}]$.

i) Any $\Sigma \mu$-algebra structure on $\mathfrak{m}$ is determined by the linear map $\operatorname{Sym}^{2}(\mathfrak{h}) \rightarrow \mathfrak{z}$, given by $\bar{x} \odot \bar{y} \mapsto x y, x, y \in \mathfrak{m}$.

ii) Starting with a linear map $\Phi: \operatorname{Sym}^{2}(\mathfrak{h}) \rightarrow \mathfrak{z}$, define $x y:=\Phi(\bar{x} \odot \bar{y}), x, y \in$ $\mathfrak{m}$. This multiplication on $\mathfrak{m}$ satisfies all conditions of Definition 7 except ii). If additionally $\mathfrak{z} \subset[\mathfrak{m}, \mathfrak{m}]$ then this multiplication defines a $\Sigma \mu$-algebra structure on $\mathrm{m}$.

iii) A Lie algebra $\mathfrak{m}$ is $\Sigma \mu$-rigid if either $\mathfrak{m}$ is perfect, or the center of $\mathfrak{m}$ is trivial.

Proof i) is a weak form of Lemma 8. ii) is trivial, while iii) is immediate from i).

As an illustration of Corollary 9 we can take $\mathfrak{m}$ to be the Heisenberg Lie algebra of dimension $2 k+1$, with basis $x_{1}, \ldots, x_{k}, y_{1}, \ldots, y_{k}, z$ such that $\left\{x_{i}, y_{i}\right\}=z$ for all $i=1, \ldots, k$ and other nontrivial brackets being zero. Choose a subset $I \subset\{1, \ldots, k\}$ and define a $\Sigma \mu$-algebra structure by

$$
\begin{aligned}
x_{i} z & =z x_{i}=y_{i} z=z y_{i}=z z=0, \\
x_{i} y_{i} & =y_{i} x_{i}= \begin{cases}z & \text { if } i \in I, \\
0 & \text { if } i \notin I,\end{cases} \\
x_{i} y_{j} & =y_{j} x_{i}=0, i \neq j .
\end{aligned}
$$

Proposition 10 Let $\operatorname{Lie}_{\Sigma \mu}(V)$ be the free Lie $\Sigma \mu$-algebra freely generated by a module $V$. Then the map $\mu^{\prime}$ from the Lemma 8 is injective and hence one has a central extension of Lie $\Sigma \mu$-algebras:

$$
0 \rightarrow \operatorname{Sym}^{2}(V) \stackrel{\mu^{\prime}}{\rightarrow} \operatorname{Lie}_{\Sigma \mu}(V) \rightarrow \operatorname{Lie}(V) \rightarrow 0 .
$$

Proof We set $\mathfrak{m}=\operatorname{Lie}_{\Sigma \mu}(V)$. Since the left adjoint of the composite functor

$$
\mathfrak{L} \mathfrak{I E} \subset \mathfrak{L} \mathfrak{B} \stackrel{\text { forget }}{\longrightarrow} \mathfrak{M O D}
$$

is the composite of corresponding left adjoint functors, we have $\mathfrak{m}_{\mathrm{Lie}}=\operatorname{Lie}(V)$. By similar reasoning $\mathfrak{m}_{\mathrm{AB}} \cong V$. For simplicity we identify these modules. For the map $\mu^{\prime}$ we have 


$$
\mu^{\prime}(u \odot v)=u v .
$$

Here $u, v \in V=\mathfrak{m}_{\mathrm{AB}}$ and $u v \in \mathfrak{m}$.

Any commutative, associative nilpotent algebra of class two can be considered as a Lie $\Sigma \mu$-algebra with trivial bracket. In particular, we can take the free such algebra generated by $V$, that is

$$
\text { Com.Ass.Nil } 2(V)=V \oplus \operatorname{Sym}^{2}(V) \text {, }
$$

where for $x, y \in V, \omega, \omega_{1} \in \operatorname{Sym}^{2}(V)$ one sets

$$
x y:=x \odot y \in \operatorname{Sym}^{2}(V), \quad x \omega=0, \quad\{x, y\}=0 \quad\{x, \omega\}=0,\left\{\omega, \omega_{1}\right\}=0 .
$$

By universal property of free Lie $\Sigma \mu$-algebras the identity map $\mathrm{Id}_{V}$ has a unique extension $c: \operatorname{Lie}_{\Sigma \mu}(V) \rightarrow$ Com.Ass.Nil $2(V)$. For any $u, v \in V$ we have

$$
c \circ \mu^{\prime}(u \odot v)=c(u v)=c(u) c(v)=u \odot v .
$$

Thus $c \circ \mu^{\prime}=\operatorname{ld}_{\mathrm{Sym}^{2}(V)}$ and the result follows.

\section{The case when 2 is invertible in $\mathrm{K}$}

In this section we assume that 2 is invertible in $\mathrm{K}$. Our goal is to prove the following result.

Theorem 11 The categories of symmetric Leibniz algebras and Lie $\Sigma \mu$-algebras are isomorphic.

This a consequence of Propositions 12 and 13 below.

Proposition 12 Let $\mathcal{L}$ be a symmetric Leibniz algebra. We put:

$$
\begin{aligned}
2\{x, y\} & =[x, y]-[y, x], \\
2 x y & =[x, y]+[y, x] .
\end{aligned}
$$

(Thus $[x, y]=\{x, y\}+x y$.) Then $\mathcal{L}$ together with operations $\{-,-\}$ and $\mu(x, y)=x y$ is a Lie $\Sigma \mu$-algebra.

Proof First we look at the bracket $\{-,-\}$. The relation $\{x, x\}=0$ is obvious. Since $[x,-]$ and $[-, x]$ are derivations, the same is true for $\{x,-\}$. Thus $\{-,-\}$ is a Lie algebra structure.

Next about the multiplication. Obviously the map $(x, y) \mapsto x y$ gives a commutative algebra structure. We have

$$
2[x, y z]=[x,[y, z]+[z, y]]=0 .
$$


Similarly

$$
2[y z, x]=[[y, z]+[z, y], x]=0 .
$$

Here we used the identity (2) and the identity (ii) of Lemma 1. It follows that

$$
2 x(y z)=[x, y z]+[y z, x]=0 .
$$

Similarly

$$
2(x y) z=[x y, z]+[z, x y]=0 .
$$

This shows that the multiplication defines a commutative and associative algebra structure of nilpotence degree two.

We have

$$
\begin{aligned}
& 4 x\{y, z\}=[x, 2\{y, z\}]+[2\{y, z\}, x] \\
& \quad=[x,[y, z]]-[x,[z, y]]+[[y, z], x]-[[z, y], x] .
\end{aligned}
$$

By the relation iii) of Lemma 1 the first and third summand gives zero, same with remaining summands. Hence $4 x\{y, z\}=0$.

Similarly

$$
4\{x y, z\}=2[x y, z]-2[z, x y]=[[x, y]+[y, x], z]-[z,[x, y]+[y, x]]=0,
$$

because of relations (2) and the identity (ii) of Lemma 1. This finishes the verification that one really obtains a Lie $\Sigma \mu$-algebra.

Proposition 13 Let $\mathfrak{m}$ be a Lie $\Sigma \mu$-algebra. We put

$$
[x, y]=\{x, y\}+x y .
$$

Then $[-,-]$ defines a symmetric Leibniz algebra structure on $\mathfrak{m}$.

Proof For $[x, y]=\{x, y\}+x y$ one has:

$$
\begin{aligned}
& {[[x, y], z]=\{[x, y], z\}+[x, y] z} \\
& \quad=\{[x, y], z\}=\{\{x, y\}, z\}+\{x y, z\}=\{\{x, y\}, z\}
\end{aligned}
$$

and hence both the right and left Leibniz identities are fulfilled. Checking (4) is not necessary, as mentioned after Definition 2. 


\section{$5 \Sigma \mu$-groups}

In this section $K=\mathbb{Z}$. The category of abelian groups is denoted by $\mathfrak{A} \mathfrak{B}$, while the category of groups is denoted by $\mathfrak{G} \Re$. It is well known that the inclusion $\mathfrak{A} \mathfrak{B} \subset \mathfrak{G} \mathfrak{R}$ has the left adjoint given by $T \mapsto T_{\mathrm{ab}}=T /[T, T], T \in \mathfrak{G} \Re$. The group $T_{\mathrm{ab}}$ is called the abelization of the group $T$.

The following is a group-theoretic version of Definition 7. Just as the latter, this is a particular case of the notion of general $\mu$-group, which we will study in [2].

Definition 14 A $\Sigma \mu$-group is a pair $(G, \mu)$, where $G$ is a group, not necessarily commutative, written additively, together with a binary operation $\mu: G \times G \rightarrow G$ such that:

i) $x y=y x$,

ii) $x(y z)=0=(x y) z$,

iii) $x y+z=z+x y$,

iv) $x(y+z)=x y+x z$.

Here $x y=\mu(x, y)$.

Obviously any commutative associative nilpotent algebra of class two is a $\Sigma \mu$ group. Also any group can be considered as a $\Sigma \mu$-group with zero multiplication.

Denote the category of $\Sigma \mu$-groups by $\mathfrak{G} \mathfrak{R}_{\Sigma \mu}$. Since any group has a trivial $\Sigma \mu$ group structure (i.e. $\mu=0$ ), we see that $\mathfrak{G} \Re \subset \mathfrak{G}_{\mathfrak{R}} \mathfrak{R}_{\Sigma \mu}$. The inclusion has a left adjoint. To describe it, we fix some obvious facts.

For a $\Sigma \mu$-group $G$, denote by $\mathrm{Z}_{\mathrm{gr}}(G)$ the center of the group $G$, that is

$$
\mathrm{Z}_{\mathrm{gr}}(G)=\{x \in G \mid x+z=z+x, \text { for all } z \in G\} .
$$

Denote by $K$ the subgroup of $G$ generated by all elements of the form $x y, x, y \in G$. By iii) $K \subset \mathrm{Z}_{\mathrm{gr}}(G)$. Thus $K$ is a central subgroup of the underlying group of $G$ and we can consider the corresponding quotient group $G_{\mathrm{gr}}=G / K$. Then the assignment $G \mapsto G_{\mathrm{gr}}$ defines the functor $\mathfrak{G} \Re_{\Sigma \mu} \rightarrow \mathfrak{G} \Re$, which is the left adjoint to the inclusion $\mathfrak{G} \mathfrak{R} \subset \mathfrak{G} \Re_{\Sigma \mu}$. We also need the group $\left(G_{\mathrm{gr}}\right)_{\mathrm{ab}}=G_{\mathrm{gr}} /\left[G_{\mathrm{gr}}, G_{\mathrm{gr}}\right]$, which is denoted by $G_{\mathrm{AB}}$ and is called the abelization of a $\Sigma \mu$-group $G$.

For $\Sigma \mu$-groups one can introduce the notion of a $\Sigma \mu$-subgroup in an obvious way. A $\Sigma \mu$-subgroup $H$ of $\Sigma \mu$-group $G$ is normal if it is a normal subgroup of the underlying group and $H G \subset H$. A $\Sigma \mu$-subgroup $H$ is central if

$$
a+x=x+a, \text { and } a x=0
$$

hold for all $x \in G$ and $a \in H$. Now, it should be clear the mining of the central extension of $\Sigma \mu$-groups. Observe that $K$ is a central $\Sigma \mu$-subgroup of $G$.

Thanks to the condition iv), the map $\lambda_{x}: G \rightarrow G$ given by $\lambda_{x}(y)=x y$ is a group homomorphism for all $x \in G$. Moreover by ii) it factors through the group $G_{\mathrm{gr}}$. Since $\operatorname{Im}\left(\lambda_{x}\right) \subset \mathrm{Z}_{\mathrm{gr}}(G)$, we see that $\lambda_{x}$ is a homomorphism into an abelian group $\mathrm{Z}_{\mathrm{gr}}(G)$ and hence it factors through the abelization $G_{\mathrm{AB}}$. It follows from the condition i) that the map $\mu$ yields a well-defined homomorphism 


$$
\operatorname{Sym}^{2}\left(G_{\mathrm{AB}}\right) \rightarrow \mathrm{Z}_{\mathrm{gr}}(G) ; \bar{x} \odot \bar{y} \mapsto x y,
$$

which we by abuse of notation will still be denoted by $\mu$. Here $\bar{x}$ denotes the class of $x \in G$ in $G_{\mathrm{AB}}$.

It is clear that $K=\operatorname{Im}(\mu)$ and hence one has a natural exact sequence of $\Sigma \mu$-groups

$$
\operatorname{Sym}^{2}\left(G_{\mathrm{AB}}\right) \stackrel{\mu}{\rightarrow} G \rightarrow G_{\mathrm{gr}} \rightarrow 0 .
$$

Moreover, $\operatorname{Im}(\mu)=K$ is a central $\Sigma \mu$-subgroup of $G$.

A group $G$ is called $\Sigma \mu$-rigid if the only $\Sigma \mu$-group structure on it is the trivial one: $x y=0, x, y \in G$. As in the case of Lie $\Sigma \mu$-algebras we have the following analogue of Corollary 9.

Corollary 15 Let $M$ be a group, $Z$ be the center of $M$ and $H=M /[M, M]$.

i) Any $\Sigma \mu$-group structure on $M$ determines a homomorphism of abelian groups $\operatorname{Sym}^{2}(H) \rightarrow Z$, given by $\bar{x} \odot \bar{y} \mapsto x y, x, y \in M$.

ii) Starting with a linear map $\Phi: \operatorname{Sym}^{2}(H) \rightarrow Z$, define $x y:=\Phi(\bar{x} \odot \bar{y}), x, y \in M$. This multiplication satisfies all conditions of Definition 7 except ii). If additionally $Z \subset[M, M]$ then this multiplication defines a $\Sigma \mu$-group structure on $\mathfrak{m}$.

iii) A group $M$ is $\Sigma \mu$-rigid if either $M$ is perfect, that is $M=[M, M]$ or the center of $M$ is trivial.

We omit the proof of this fact, since it goes along the same lines as the proof of Corollary 9.

Here is some examples based on Part ii) of Corollary 15. Take the dihedral group $D_{2 n}$ of order $4 n$ with generators $x$ and $y$, such that $2 n x=0=2 y$ and $y+x+y=(2 n-1) x$. One easily sees that there exists a unique $\Sigma \mu$-algebra structure such that $x y=n x$, $x x=0=y y$. As another example, one can introduce a nontrivial $\Sigma \mu$-group structure on the Heisenberg group, similar to one which we described for Heisenberg Lie algebra. Details are left to the interested readers.

Denote by $\mathbb{Z}[S]$ the free abelian group generated by a set $S$. Similarly, denote by $\operatorname{Gr}[S]$ (resp. $\operatorname{Gr}_{\Sigma \mu}[S]$ ) the free group (resp. free $\Sigma \mu$-group) generated by $S$. In these notations we have

Lemma 16 One has the following central extension of $\Sigma \mu$-groups.

$$
0 \rightarrow \operatorname{Sym}^{2}(\mathbb{Z}[S]) \stackrel{\mu}{\rightarrow} \operatorname{Gr}_{\Sigma \mu}[S] \rightarrow \operatorname{Gr}[S] \rightarrow 0
$$

Proof For $G=\operatorname{Gr}_{\Sigma \mu}[S]$, one has $G_{\mathrm{gr}}=\operatorname{Gr}[S]$ and $G_{\mathrm{AB}}=\mathbb{Z}[S]$, by the adjoint functor argument (see the proof of Proposition 10). Thus we can use the exact sequence (6). We only need to show injectivity of $\mu$.

Any commutative, associative nilpotent ring of class two can be considered as a $\Sigma \mu$-group. In particular, we can take the free such a ring generated by $V=\mathbb{Z}[S]$, that is

$$
\text { Com.Ass.Nil } 2(\mathbb{Z}[S])=\mathbb{Z}[S] \oplus \operatorname{Sym}^{2}(\mathbb{Z}[S])
$$


By the universal property of free $\Sigma \mu$-groups the identity map $\operatorname{ld}_{S}$ has a unique extension

$$
c: \operatorname{Gr}_{\Sigma \mu}[S] \rightarrow \text { Com.Ass.Nil } 2(\mathbb{Z}[S])
$$

We have

$$
c \circ \mu(x \odot y)=c(x y)=c(x) c(y)=x \odot y
$$

for all $x, y \in S$. Thus $c \circ \mu=\operatorname{ld}_{\mathrm{Sym}^{2}(\mathbb{Z}[S])}$ and the result follows.

\section{Lie $\Sigma \mu$-groups}

In this section $K=\mathbb{R}$. Denote by $\mathbf{L}$ the canonical functor from the category of Lie groups to the category of finite dimensional Lie algebras. Recall that the restriction of $\mathbf{L}$ to the subcategory of simply connected Lie groups is an equivalence of categories [15, Theorem 2, p.152].

As Lie groups are smooth analogues of discrete groups, the following is a smooth analogue of $\Sigma \mu$-groups.

Definition 17 A Lie $\Sigma \mu$-group is a smooth manifold $M$, equipped with a structure of $\Sigma \mu$-group, such that the maps

$$
\mu,+: M \times M \rightarrow M, \quad-: M \rightarrow M
$$

are smooth.

Now we extend the functor $\mathbf{L}$ to Lie $\Sigma \mu$-groups.

Let $M$ be a Lie $\Sigma \mu$-group with $\Sigma \mu$-structure $\mu(x, y)=x y$. Denote by $K$ the closure of the subgroup generated by elements of the form $x y, x, y \in M$. Then $G=M / K$ is also a Lie group. Denote by $G^{\prime}$ the closure of the commutator $[G, G]$. Then $H:=G / G^{\prime}$ is an abelian Lie group.

Since $(x y) z=0=x(y z)$ holds for all $x, y, z \in M$ and $\mu$ is smooth, it follows that $\mu(K, x)=0=\mu(x, K)$ for all $x \in M$. Based on this and use the identity (iv) of Definition 14, we see that $\mu$ factors through the map $G \times G \rightarrow Z$, where $Z$ is the center of $M$. By abuse of notations we still denote this map by $\mu$. As we have seen in Sect. 5, $\mu$ vanished on the commutators $\mu([G, G], x)=0=\mu(x,[G, G])$. Since $\mu$ is smooth it vanishes on $G^{\prime}$ yielding a smooth map $\mu: H \times H \rightarrow Z$ which is a homomorphism of abelian Lie groups with respect to each variables. It follows that it defines a bilinear multiplication on corresponding Lie algebras $\mathbf{L}(H) \times \mathbf{L}(H) \rightarrow \mathbf{L}(Z)$ and hence the multiplication on $\mathbf{L}(M)$ via

$$
\mathbf{L}(M) \times \mathbf{L}(M) \rightarrow \mathbf{L}(G) \times \mathbf{L}(G) \rightarrow \mathbf{L}(H) \times \mathbf{L}(H) \rightarrow \mathbf{L}(Z) \subset \mathbf{L}(M) .
$$

It follows from the part ii) of Corollary 9 that this is in fact a Lie $\Sigma \mu$-algebra structure on $\mathbf{L}(M)$. 
Thus we constructed the functor

$$
\{\text { Lie } \Sigma \mu \text {-groups }\} \stackrel{\mathbf{L}}{\rightarrow}\{\text { Lie } \Sigma \mu \text {-algebras }\} \text {. }
$$

Theorem 18 The functor $\mathbf{L}$ yields an equivalence between the category of simply connected Lie $\Sigma \mu$-groups and finite dimensional Lie $\Sigma \mu$-algebras.

Proof We construct the functor

$$
\{\text { f.d. Lie } \Sigma \mu \text {-algebras }\} \stackrel{\mathbf{R}}{\rightarrow}\{\text { simply connected Lie } \Sigma \mu \text {-groups }\}
$$

as follows.

Let $\mathfrak{m}$ be a Lie $\Sigma \mu$-algebra. Denote by $\mathfrak{z}$ the center of the underlying Lie algebra of $\mathfrak{m}$. We also set $\mathfrak{g}:=\mathfrak{m}_{\text {Lie }}$ and $\mathfrak{h}:=\mathfrak{m}_{A B}=\mathfrak{g}_{\mathrm{ab}}$. Then we have an exact sequence

$$
\mathfrak{h} \otimes \mathfrak{h} \stackrel{\mu}{\rightarrow} \mathfrak{m} \stackrel{p}{\rightarrow} \mathfrak{g} \rightarrow 0
$$

Moreover, $\mathfrak{a}:=\operatorname{Im}(\mu) \subset \mathfrak{z}$ is a central subalgebra of the underlying Lie algebra of $\mathfrak{m}$.

From now, we assume that $\mathfrak{m}$ is finite dimensional. Obviously $\mathfrak{g}$ is also finite dimensional. By mentioned equivalence of categories there are simply connected Lie groups $M$ and $G$ and a homomorphism of Lie groups $\rho: M \rightarrow G$ such that $\mathbf{L}(M)=\mathfrak{m}$, $\mathbf{L}(G)=\mathfrak{g}$ and $\mathbf{L}(\rho)=p$. Obviously this can be done functorially with respect of $\mathfrak{m}$. By [8, Corollary, p.40 ] the center $Z$ of $M$ is a normal Lie subgroup and $\mathbf{L}(Z)=\mathfrak{z}$.

We set $A=\operatorname{Ker}(\rho)$. Since $p$ is surjective, $\rho$ is also surjective, see part (iii) of [14, Theorem 2, p.76]. Thus we have a short exact sequence of Lie groups

$$
0 \rightarrow A \rightarrow M \stackrel{\rho}{\rightarrow} G \rightarrow 0
$$

By the part (ii) of [14, Theorem 2, p.76] we have $\mathbf{L}(A)=\mathfrak{a} \subset \mathfrak{z}$. Hence $A$ is a central subgroup of $M$. So $A \subset Z$. Since $\rho$ is a fibration with fibre $A$ the homotopy exact sequence shows that $A$ is connected.

Since $G$ is simply connected, the commutator $[G, G]$ is a closed and connected subgroup of $G$ with $\mathbf{L}([G, G])=[\mathfrak{g}, \mathfrak{g}]$, see [8, Theorem 5.1]. It follows that $H:=$ $G /[G, G]$ is a well-defined abelian Lie group with $\mathbf{L}(G /[G, G])=\mathfrak{h}$. The map $G \rightarrow H$ is a fibration with connected fibre $[G, G]$. It follows that $H:=G /[G, G]$ is a simply connected, abelian Lie group. Thus the canonical map exp $: \mathfrak{h} \rightarrow H$ is an isomorphism. We can now form the diagram

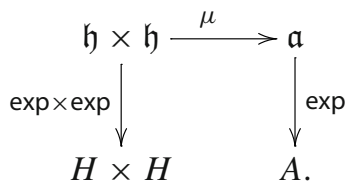

Since the left arrow is an isomorphism of Lie groups, we obtain a smooth map $H \times$ $H \rightarrow A \subset Z$. Now we can take the composite map 


$$
M \times M \rightarrow G \times G \rightarrow H \times H \rightarrow Z \subset M,
$$

to obtain the multiplication map in $M$. By Part ii) of Corollary 15 in this way we obtain a $\Sigma \mu$-group structure on $M$. This finishes the construction of the functor $\mathbf{R}$.

Take a simply connected Lie $\Sigma \mu$-group $M$. Recall that $K$ was defined as the closure of the subgroup generated by $x y, x, y \in M$. It follows that $K$ is connected and hence $G=M / K$ is also simply connected. Now, comparing the constructions we see that $\mathbf{R L}(M) \cong M$.

Conversely, if $\mathfrak{m}$ is a finite dimensional $\Sigma \mu$-algebra, then $\mathfrak{a}$, which is defined as the subspace of $\mathfrak{m}$ generated by elements of the form $x y, x, y \in \mathfrak{m}$ coincides with the subgroup generated by the same elements. This follows from the (bi)linearity of the product. Since $A$ is abelian, the exponential map exp : $\mathfrak{a} \rightarrow A$ is epimorphism. From this follows that the subgroup $K \subset M=\mathbf{R}(\mathfrak{m})$ and $A$ coincide and $\mathbf{L R}(\mathfrak{m}) \cong \mathfrak{m}$.

Corollary 19 The category of finite dimensional symmetric Leibniz algebras is equivalent to the category of simply connected Lie $\Sigma \mu$-groups.

Proof This is a direct consequence of Theorems 11 and 18.

\section{Interpretation in language of algebraic theories}

The algebraic theories corresponding to Lie, Leibniz and symmetric Leibniz Kalgebras are denoted respectively by $\mathscr{L} \mathscr{I} \mathscr{E} \mathrm{K}, \mathscr{L} \mathscr{B} \mathrm{K}$ and $\mathscr{S} \mathscr{L} \mathscr{B} \mathrm{K}$.

We refer to [1] for the notion of (linear) extension of algebraic theories. Comparing the definitions we see that Proposition 5 can be restated as

Lemma 20 One has a linear extension of algebraic theories:

$$
0 \rightarrow \Gamma^{2}\left((-)_{a b}\right)+\rightarrow \mathscr{S} \mathscr{L} \mathscr{B}_{\mathrm{K}} \rightarrow \mathscr{L} \mathscr{I} \mathscr{E} \mathrm{K} \rightarrow 0
$$

Moreover if 2 is invertible in $\mathrm{K}$, this extension splits.

Recall that the functor Rings $\rightarrow$ Algebraic Theories which sends the ring $R$ to the theory of right $R$-modules is full and faithful. Because of this we identify rings with their corresponding theories of modules.

Let $\mathscr{C O} \mathscr{M}_{\mathrm{K}}(2)$ denote the algebraic theory of nilpotent commutative associative $\mathrm{K}$-algebras of class 2 , that is algebras obeying the identities

$$
x y=y x, \quad(x y) z=0 .
$$

Then one has a split linear extension of algebraic theories (compare [1])

$$
0 \rightarrow \operatorname{Sym}^{2}\left((-)_{a b}\right)+\rightarrow \mathscr{C O O} \mathscr{M}_{\mathrm{K}}(2) \rightarrow \mathrm{K} \rightarrow 0
$$

If 2 is invertible in $\mathrm{K}$, then $\Gamma^{2}=\mathrm{Sym}^{2}$ and we get the following 
Proposition 21 If 2 is invertible in $\mathrm{K}$, then the diagram

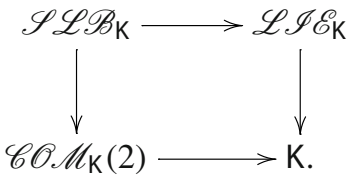

is the pull-back diagram in the category of algebraic theories.

Quite similarly, if one denotes by $\mathscr{G} \mathscr{R}$ (resp. $\mathscr{G} \mathscr{R}_{\Sigma \mu}, \mathscr{A} \mathscr{B}$ ) the algebraic theory of groups (rep. $\Sigma \mu$-groups, abelian groups), then as an immediate corollary of Lemma 16 we obtain the following fact

\section{Proposition 22 The diagram}

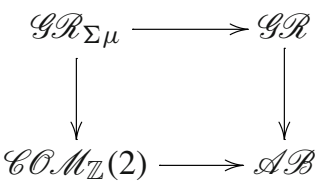

is the pull-back diagram in the category of algebraic theories.

Open Access This article is distributed under the terms of the Creative Commons Attribution 4.0 International License (http://creativecommons.org/licenses/by/4.0/), which permits unrestricted use, distribution, and reproduction in any medium, provided you give appropriate credit to the original author(s) and the source, provide a link to the Creative Commons license, and indicate if changes were made.

\section{References}

1. Jibladze, M., Pirashvili, T.: Cohomology of algebraic theories. J. Algebra 137, 253-296 (1991)

2. Jibladze, M., Pirashvili, T.: Leibniz algebras and $\mu$-groups (In preparation)

3. Loday, J.-L.: Cyclic Homology. Grundlehren der Mathematischen Wissenschaften, vol. 301, p. xviii+454. Springer, Berlin (1992). (ISBN:3-540-53339)

4. Loday, J.-L.: Une version non commutative des algébres de Lie: les algébres de Leibniz. Enseign. Math. (2) 39(3--4), 269-293 (1993)

5. Loday, J.-L.: Algebraic K-theory and the conjectural Leibniz K-theory. Special issue in honor of Hyman Bass on his seventieth birthday. Part II. K-Theory 30(2), 105-127 (2003)

6. Loday, J.-L., Pirashvili, T.: Universal enveloping algebras of Leibniz algebras and (co)homology. Math. Ann. 296(1), 139-158 (1993)

7. Loday, J.-L., Pirashvili, T.: Leibniz representations of Lie algebras. J. Algebra 181(2), 414-425 (1996)

8. Onishchik, A.L., Vinberg, E.B.: Foundation of Lie theory. 1-96. In: Gorbatsevich, V.V., Onishchik, A.L., Vinberg, E.B. (eds.) Foundations of Lie Theory and Lie Transformation Groups. Encyclopedia of Mathematical Sciences, vol. 20, p. vi+235. Springer, Berlin (1993). (ISBN: 3-540-61222-X)

9. Ronco, M.: Unpublished notes (1995)

10. Pirashvili, T.: On Leibniz homology. Ann. Inst. Fourier. 44(2), 401-411 (1994)

11. Pirashvili, T.: Introduction to functor homology. In Rational representations, the Steenrod algebra and functor homology, Panor. Synthéses, 16. Société Mathématique de France, Paris, pp. 1-26 (2003)

12. Pirashvili, T.: On strongly perfect lie algebras. Commun. Algebra. 41, 1619-1625 (2013) 
13. Pirashvili, T.: A subcomplex of Leibniz complex. arXiv:1904.00121

14. Procesi, C.: Lie Groups. An Approach Through Invariants and Representations. Univesitext, p. xxiv+596. Springer, Berlin (1967)

15. Serre, J.-P.: Lie Algebras and Lie Groups. 1964 Lectures Given at Harvard University. Lecture Notes in Mathematics, vol. 1500, p. viii+168. Springer, Berlin (2006)

Publisher's Note Springer Nature remains neutral with regard to jurisdictional claims in published maps and institutional affiliations. 